\title{
Follow-up of Adrenal Incidentalomas - are we overdoing it? Follow-up of 145 patients from a single centre
}

\author{
Dr E Austin, Dr B Miller, Sister L. Shepherd, Dr A Bates \\ Department of Diabetes and Endocrinology, Heart of England Foundation Trust
}

\section{INTRODUCTION}

The core objectives when investigating adrenal incidentalomas are to exclude functionality and malignancy via radiological and biochemical investigations. Routinely, these lesions are then followed-up with repeat investigations in case of progression as per the AACE guidelines ${ }^{1}$.

Several studies have suggested that this is unnecessary given the low conversion rates to functional or malignant lesions ${ }^{2}$. Current guidelines for monitoring adrenal incidentalomas are therefore under review by the $\mathrm{ESE}^{3}$.

Following research presented in 2015 , we suggested that in asymptomatic patients with imaging features consistent with a benign adenoma, further investigation was unrewarding ${ }^{4}$. To support this conclusion, further radiological data for the same cohort was collected to assess the frequency of conversion of presumed benign lesions to malignant ones.

\section{OBJECTIVES}

This study was performed to:

1. Review the incidence of functional or malignant lesions detected as adrenal incidentalomas

2. Assess the frequency of progression of a benign adenoma to a potentially malignant lesion

3. For those patients with repeat biochemistry, to review the risk of becoming functional when initially non-functional

\section{METHODS}

145 consecutive cases of adrenal incidentalomas referred to Endocrinology from January 2013 to January 2015 were reviewed.

\section{Data collected:}

- Age, gender, blood pressure

- Imaging results of first and last scan

- Biochemistry results (plus repeat results if available)

- Time interval between first and last scan

- Diagnosis

- Outcome

\section{Cohort Demographics:}

- Mean age 65 years (21-95)

- $M: F=51: 94$

The incidentalomas were then categorised as benign adenomas if they were $<20 \mathrm{HU}$ (on plain CT) and $<4 \mathrm{~cm}$ in size; all others were deemed indeterminate or malignant.

\section{RESULTS}

Figure 1: Summary of outcomes, diagram modified from ESE Guideline ${ }^{3}$

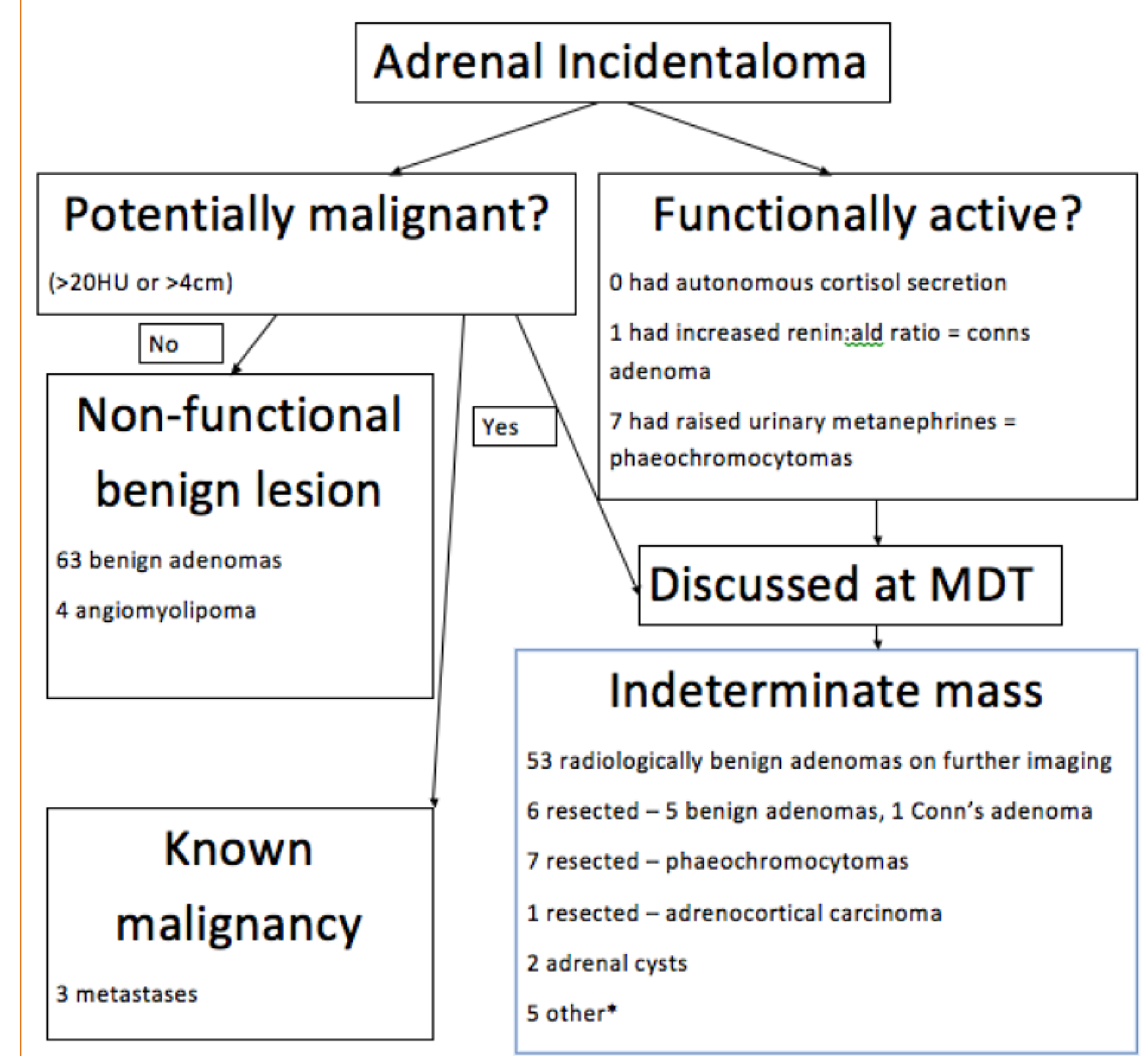

$$
\begin{array}{|l|l}
\hline \text { Adr } \\
\hline \text { Con } \\
\hline \text { Ma } \\
\hline \text { Pha } \\
\hline \text { ACC } \\
\hline \text { Me } \\
\hline \text { Oth } \\
\hline
\end{array}
$$

Benig$$
\begin{array}{|l}
\text { Adren } \\
\hline \text { Angio } \\
\hline
\end{array}
$$

\section{Angi}$$
\text { Malignant }
$$$$
\text { (a) }
$$

Figure 2: Summary of diagnoses

(20llow-up

\section{What was the frequency of progression of benign to possibly malignant lesions?}

- Of the 63 patients whose detection scan was characteristically benign, 45 (71\%) had further imaging at a median time interval of 13 months. None had progressed.

- 74 patients had indeterminate detection scans. Of these, 65 (88\%) had repeat scans at a median time interval of 8 months. The outcomes of the indeterminate lesions are detailed below:

$\rightarrow 59$ patients had benign adenomas, of which 58 had repeat scans ( 1 was resected as $>7 \mathrm{~cm}$ ) at a median time interval of 7 months. 54 were characterised as benign on repeat imaging. 2 became larger and 2 remained indeterminate so these were resected and histologically confirmed as benign adenomas.

$\rightarrow 7$ patients had phaeochromocytomas -2 of which were resected based on the initial scan, and the remaining 5 were resected following further characteristic imaging. 2 had increased in size at a median time interval of 8 months. All had raised urinary metanephrines.

$\rightarrow$ There was a solitary ACC which had not increased in size after 10 months, however was characteristically malignant on further imaging.

- All 3 metastatic lesions had increased in size on repeat imaging at a median time interval of 5 months. All patients had a known history of malignancy.

\section{Do non-functional benign lesions progress to functional lesions?}

- In the cohort of benign lesions (see figure 2), mean duration of time from initial scan to latest biochemistry was 5 months, during which $99 \%$ of incidentalomas remained non-functional.

-28 patients (22\%) in this subgroup had repeat biochemistry; none progressed from non-functional to functional lesions.

\section{$10 H U$ vs $20 H U$ when defining benign lesions?}

Based on the data collected, the sensitivity and specificity of using either $10 \mathrm{HU}$ or $20 \mathrm{HU}$ on a plain film CT to define a lesion as benign are shown in figure 3. No malignant lesions had a density of less than $20 \mathrm{HU}$.
Figure 3: Sensitivity and specificity values

\begin{tabular}{|l|lc|}
\hline $\begin{array}{l}\text { Plain film } \\
\text { CT density }\end{array}$ & Sensitivity & Specificity \\
\hline$<10 \mathrm{HU}$ & $100 \%$ & $41 \%$ \\
\hline$<2 \mathrm{HU}$ & $100 \%$ & $52 \%$ \\
\hline
\end{tabular}

\section{CONCLUSIONS}

- There is no evidence that radiologically benign lesions progress to functional or malignant lesions. Therefore, it is not necessary to repeat imaging if the detection scan is considered benign.

- For indeterminate images, if repeat imaging is characteristic of a benign adenoma, no further imaging is needed.

- A cut off of $<20 \mathrm{HU}$ on plain CT to define benign lesions may improve specificity without compromising sensitivity, therefore avoiding excessive investigation of benign lesions. 